\title{
PIECEWISE POLYNOMIALS AND THE FINITE ELEMENT METHOD ${ }^{1}$
}

\author{
BY GILBERT STRANG
}

Thirty years ago, Courant gave a remarkable lecture to this Society. My talk today is more or less a progress report on an idea which he described near the end of that lecture. There are a lot of people in this city, and a few in this room, who worked very closely with Courant--but the idea I am talking about came to fruition in a different and more unexpected way.

To begin with, his idea was forgotten. Perhaps you have forgotten it too; it had to do with approximation by piecewise polynomials, and I will try to explain it properly in a moment. Ten years later Pólya made a very similar suggestion [3], [4], without reference to Courant's lecture. At the same time, and independently, Synge did exactly the same thing [10]. Meanwhile Schoenberg had written the paper [5] which gave birth to the theory of splines-again proposing that, for approximation and interpolation, the most convenient functions were piecewise polynomials.

Certainly there was an idea whose time was coming. When it finally came, fifteen years after Courant's lecture, it developed into what is now the most powerful technique for solving a large class of partial differential equations--the finite element method. The only sad part is that virtually the whole development took place as if Courant had never existed. It is like the story of Romulus and Remus (I think); in this case, the wolves who eventually took care of the orphan happened to be structural engineers. $^{2}$ They needed a much better technique for the solution of complicated elliptic systems, and in numerical analysis the algorithms which survive and mature are those which are needed. We want to describe this finite element method, and then at the end to propose an open problem; its interest may be more algebraic-combinatorial than practical, but it is directly suggested by the construction of finite elements.

Prior to Courant, the usual approximating functions were sines and cosines, or Bessel functions, or Legendre polynomials. For a simple problem on a regular domain, these are still completely adequate; their approximation properties are well known, and integrations are simplified

AMS(MOS) subject classifications (1970). Primary 35J20, 41A15, 41A25, 65N30.

Key words and phrases. Partial differential equations, finite element method, approximation, piecewise polynomials.

${ }^{1}$ The preparation of this lecture, delivered to the American Mathematical Society in New York City on April 20, 1973, was supported by the National Science Foundation (P22928).

${ }^{2}$ I hope they will forgive the analogy. 
by orthogonality. On an irregular domain (an airplane, for example, or a human skull-both of whose eigenfrequencies have now been computed), the situation is completely different; these functions are virtually useless. What is needed is a class of approximants which can be defined, and made to satisfy boundary conditions, on domains of any reasonable shape.

Courant's idea was very simple. He triangulated the domain and introduced the space of continuous piecewise linear functions: $v=$ $a+b x+c y$ within each triangle. The function $v$ is uniquely determined by its values at the vertices of the triangulation; equivalently, the space has a convenient basis. If the vertices are $z_{1}, \ldots, z_{N}$, and a linear $\varphi_{j}$ is determined in each triangle by $\varphi_{j}\left(z_{i}\right)=\delta_{i j}$, then any element of Courant's space can be expanded as a combination of the $\varphi_{j}$ :

$$
v(x, y)=\sum_{1}^{N} v\left(z_{j}\right) \varphi_{j}(x, y) .
$$

Notice that $\varphi_{j}$ is automatically continuous across the edges between triangles. It is linear along such an edge, and is therefore determined by its values at the two vertices at the ends; these are held in common by the two triangles, and continuity is assured.

This basis is called local, because each $\varphi_{j}$ is nonzero only in those triangles of which $z_{j}$ is a vertex; the graph of $\varphi_{j}$ is just a pyramid, with its apex over the vertex $z_{j}$. The basis is not orthogonal, but nevertheless almost all of the inner products $\iint \varphi_{j} \varphi_{k}$ are zero; unless $z_{j}$ and $z_{k}$ belong to the same triangle, the integrand vanishes identically. Therefore the matrices which arise in the finite element method are sparse and are welcomed by the computer.

This space was later rediscovered by the engineers, but at first in an indirect way; their intuition took them directly to the applications which we describe below, and it was only gradually that their equations were recognized as instances of the Rayleigh-Ritz principle applied to Courant's trial functions. These piecewise linear functions are still useful in complicated nonlinear problems; probably they remain the most frequently used finite elements. For solving the standard equations of linear elasticity, however, they have definitely been superseded. In fact, we trace the real beginning of the finite element method to the time when Courant's space was actually used on a large scale, and its defects became clear. It was these defects which forced a further development of the underlying idea. We mention three of the worst:

(i) linear polynomials permit only a crude approximation of the true solution;

(ii) the derivatives of Courant's elements are discontinuous at the boundaries between triangles; 
(iii) a curved domain will be inaccurately represented by a union of triangles (or squares, on which the trial functions become bilinear: $a+b x+c y+d x y)$.

The problem is therefore to construct spaces which are as simple as Courant's, or nearly so, and at the same time free of these drawbacks. Dozens of such "finite element spaces" have been proposed; we take up the three defects in order.

(i) The basic order of approximation is improved by increasing the degree of the polynomials, for example to continuous piecewise quadratics $\left(a+b x+c y+d x^{2}+e x y+f y^{2}\right.$ in each triangle). To be useful in computation, however, this space must have a local basis (not every space does). Its construction is the first important step beyond Courant's: add to the vertices a further set of nodes placed at the midpoints of the edges. Now, there are six nodes for every triangle, and the rule $\varphi_{j}\left(z_{i}\right)=\delta_{i j}$ determines a unique quadratic. It is automatically continuous between triangles, because the three nodes on an edge (two vertices and one midpoint) determine the quadratic along that edge. Again, each element in the space can be written as $v=\sum v\left(z_{j}\right) \varphi_{j}$.

The continuous piecewise cubics also admit a local basis. There are ten parameters in a cubic and therefore ten nodes for each triangle, arranged like bowling pins: one at each vertex, two more along each edge, and one at the centroid. A similar construction applies to $C^{0}$ polynomials of degree $p$ in any number of variables.

It is essential to know the dimension of these spaces. This means counting the nodes, and we need the observation that vertices, triangles, and edges occur in the ratio $1: 2: 3$.

Proof. Each interior vertex accounts for 360 degrees, and each triangle for 180; thus, there are about twice as many triangles as vertices. If we count each edge as half in one triangle and half in its neighbor, that gives two triangles for three edges. (Or introduce one new vertex within a triangle; there are three new edges and a net gain of two triangles. The $1: 2: 3$ rule needs a slight correction from the Euler-Poincaré formula, because of the outer boundary of the domain. In fact, according to the Euler polyhedron formula (Tutte [11]), $\alpha$ interior and $\beta$ boundary vertices produce $2 \alpha+\beta-2$ triangles and $3 \alpha+2 \beta-3$ edges.) If $V$ is the number of vertices, then Courant's space has dimension $V$, the quadratic space has dimension $V+E \sim 4 V$, and the cubic space $V+2 E+T \sim$ $9 V$. With degree $p$ in $n$ variables the coefficient increases to $p^{n}$; this factor governs the number of parameters in the approximation and in practice it is rarely allowed to reach as high as 10 .

(ii) It is surprisingly hard to piece together polynomials in such a way that not only $v$ but also its normal derivative $v_{n}$ is continuous between 
triangles. Such a construction is officially required for biharmonic problems $\Delta^{2} u=f$; otherwise the second derivatives of $v$ involve delta functions and the associated energy $\iint|\Delta v|^{2} d x d y$ becomes infinite. These nonconforming elements, with infinite energy, are nevertheless frequently used in engineering computations. All the rules of the Rayleigh-Ritz method are broken, but the answers are sometimes very good (and sometimes not). We return below to these "variational crimes." In three variables it appears that $C^{1}$ continuity between tetrahedra is first achieved by finite element polynomials of degree 9 . Such a construction is out of the question in practice, and the use of an illegal element is inevitable.

In two variables $C^{1}$ continuity is possible for quintic polynomials. There are 21 coefficients in a quintic and 18 conditions will be specified by the values of $v, v_{x}, v_{y}, v_{x x}, v_{x y}$, and $v_{y y}$ at the vertices; the vertices become six-fold nodes. The other three conditions come from matching the normal derivatives $v_{n}$ at the midpoints of the edges. Along an edge between triangles, we find the same quintic from both sides, since three conditions are shared at each end of the edge $-v$ and its tangential derivatives $v_{t}$ and $v_{t t}$, which are computable from the six parameters at the vertex. Also the normal derivative $v_{n}$ is continuous across the edge; it is a quartic and is uniquely determined by its value at the midpoint, together with $v_{n}$ and $v_{n t}$ at each end. All these values are common to the two quintics which meet at the edge.

These quintics are of class $C^{1}$, with $C^{2}$ continuity at the vertices; this characterizes the space. To find a basis, we have to generalize the rule $\varphi_{j}\left(z_{i}\right)=\delta_{i j}$, which applied when only function values were specified at the nodes; the space now involves the derivative $\partial / \partial n$ at the edge midpoints, and six derivatives $D_{j}=I, \partial / \partial x, \ldots, \partial^{2} / \partial y^{2}$ at the vertices. Each of these nodal parameters, specified by a node $z_{j}$ and a derivative $D_{j}$, corresponds to a basis function $\varphi_{j}$. In other words, for each pair $\left(z_{j}, D_{j}\right)$ we have $D_{j} \varphi_{j}\left(z_{j}\right)=1$; and the other nodal parameters of $\varphi_{j}$ are $D_{i} \varphi_{j}\left(z_{i}\right)=$ 0 . This means six basis functions for each vertex and one for each midpoint; since there are three times as many edges as vertices, the dimension is $9 \mathrm{~V}$.

On a square mesh there is a much simpler $C^{1}$ element-cubic in each variable separately, with 16 coefficients in the polynomial and $v, v_{x}, v_{y}$, and $v_{x y}$ as nodal parameters at the four vertices.

(iii) The third difficulty with Courant's space is the change from a smooth domain $\Omega$ to a triangulated polygon. We have studied elsewhere [8] the effect on the solution; it is always serious near the boundary $\partial \Omega$, and it will be dominant also in the interior if we improve the underlying polynomials to quadratics or cubics. The same problem is present for finite difference approximations; it is hard to find any reasonable 
difference formula near a curved boundary, and nearly impossible to maintain high accuracy. For finite differences, this difficulty is still unresolved; its solution for finite elements has given them a tremendous advantage.

In the theory of partial differential equations, boundaries are straightened by a local change of coordinates. Here we do the same, mapping curved triangles in the $x-y$ plane into straight triangles in $\xi-\eta$. The problem is to keep the coordinate changes computable, and Irons found the perfect solution: Use the same class of piecewise polynomials for the coordinate transformations $x(\xi, \eta), y(\xi, \eta)$ as for the approximations to $u(\xi, \eta)$. Such transformations are called isoparametric and the interelement continuity of finite element polynomials assures that neighboring elements in $\xi-\eta$ remain neighbors in $x-y$. The change of coordinates is determined purely by the movement of the nodes. With piecewise quadratic or cubic approximations to the boundary, the change of domain error becomes negligible, and an entirely new class of applications is made possible.

Approximate solution of partial differential equations. We want to explain briefly how these approximating spaces are used. The finite element method is based on variational principles rather than differential equations; in many applications an extremal principle is the primary physical law, and the Euler equation is only a secondary consequence. Therefore the shift toward direct approximation of the variational problem and away from difference approximation of the Euler equation is not surprising. The distinction is especially important for natural boundary conditions, which need not be satisfied by the trial functions in the extremal problem. The real theme of Courant's lecture (the finite element method was only an afterthought) was to perturb variational principles by a "penalty" term in order that all boundary conditions should become natural; this is justified by the theory, but does not seem to have become popular in engineering calculations. The essential (Dirichlet) conditions are of lower order and much less difficult to satisfy.

Suppose the problem is to minimize a quadratic functional $J(v)=$ $a(v, v)-2 b(f, v)$ over a space $V$ of admissible functions-normally $V$ contains those functions with finite strain energy, $a(v, v)<\infty$, which satisfy the essential boundary conditions.

EXAMPLE. $a(v, v)=\iint v_{x}^{2}+v_{y}^{2}, b(f, v)=\iint f v, V=\mathscr{H}_{0}^{1}$ (essential condition: $u=0$ on $\partial \Omega$ ). The Euler equation for the minimizing $u$ is linear and selfadjoint: $-\Delta u=f$ in $\Omega, u=0$ on $\partial \Omega$. Its weak form is

$$
\iint u_{x} v_{x}+u_{y} v_{y}=\iint f v,
$$


or $a(u, v)=b(f, v)$ for $v$ in $V$. This is the engineer's equation of virtual work.

The Rayleigh-Ritz idea almost suggests itself-to choose a finitedimensional subspace $S \subset V$, and minimize the functional $J(v)$ over $S$. The idea has a long history, but with hand computation the space $S$ had to be very small and the approximation correspondingly poor. I suppose it was the computer which demanded a new construction; the dimension of $S$ could be large, if its basis were kept sufficiently simple. This means piecewise polynomials and the finite element method. Let the trial functions, as constructed earlier, be $v=\sum q_{j} \varphi_{j}$. Then the functional to be minimized becomes a quadratic in the unknowns $q=\left(q_{1}, \ldots, q_{N}\right)$ :

$$
J(v)=a\left(\sum q_{j} \varphi_{j}, \sum q_{j} \varphi_{j}\right)-2 b\left(f, \sum q_{j} \varphi_{j}\right)=q^{T} K q-2 q^{T} F .
$$

The entries of the matrix $K$ are the energy inner products $a\left(\varphi_{j}, \varphi_{k}\right)$, and the vector $F$ has components $b\left(f, \varphi_{k}\right)$. The optimal coefficients $Q_{j}$, those which minimize this quadratic, are determined by the linear system $K Q=F$. These $Q_{j}$ are the nodal values of the Ritz-finite element approximation $u_{S}=\sum Q_{j} \varphi_{j}$, which minimizes $J(v)$ over $S$. This is therefore what the computer has to do: given the locations of the nodes, it connects the nodal values of $v$ (or more generally the nodal parameters $D_{j} v$ ) to the coefficients of the polynomial in the triangle, integrates to find $K$ and $F$, and then solves $K Q=F$.

Convergence of the finite element method. Numerical analysis is partly pure mathematics and partly applied: a convergence proof is a purely theoretical result, but it becomes important and alive only when the underlying algorithm is successful in practice. The applications govern the theory, over the long term. This is not the place to explain the finite element subroutines which compute the stiffness matrix $K$ and the load vector $F$; what matters is that they are very fast and the algorithm is a success (of course it may not be immortal). Apparently the power of the computer is applied to the formulation of the discrete problem more effectively than in any previous technique; the solution $Q$ is ordinarily still computed by an algorithm of Gauss.

The convergence of the approximation $u_{S}$ to $u$ is governed by a single fundamental principle: if the energy form $a(v, v)$ is positive definite, then in the intrinsic norm $\|v\|^{2}=a(v, v)$, the approximation $u_{S}$ is the projection of the true solution $u$ onto the subspace $S$. Therefore,

$$
\left\|u-u_{S}\right\|=\min _{v \in S}\|u-v\|
$$

and convergence becomes a problem in approximation theory-to estimate the distance between $u$ and $S$. Such an estimate will depend both 
on the properties of $u$ (its smoothness is predicted by the theory of elliptic equations) and on the degree of the polynomials in $S$.

Elsewhere [6], [9] we have given a fuller discussion of approximation by piecewise polynomials, to which a great many authors have contributed. ${ }^{3}$ Here we propose only to summarize the results, under two simplifying assumptions:

(i) $S$ contains all polynomials of degree less than or equal to $p$ ( $p=1$ for the linear and bilinear spaces, $p=2$ for quadratics, . . );

(ii) the lengths of all edges lie between $\alpha h$ and $h$.

Then for any $u$ with $p+1$ derivatives, there is a $v$ in the space $S$ such that

$$
\|u-v\|_{j} \leqq C h^{p+1-j}\|u\|_{p+1} .
$$

When these represent maximum norms of the derivatives of order $j$ and $p+1$, respectively, a simple choice of the approximating $v$ is found by interpolating the nodal values of $u$, and the proof follows from Taylor series expansions. In mean-square (Sobolev) norms, the proof is more difficult-and more important, if the strain energy is itself a sum of squares of $m$ th derivatives. In this strongly elliptic case, when the intrinsic norm satisfies $\|v\| \geqq c\|v\|_{m}$, the rate of convergence follows immediately from (1) and (2): $u_{S}$ is closer to $u$ than the interpolating function $v$, and therefore

$$
\left\|u-u_{S}\right\|=O\left(h^{p+1-m}\right) .
$$

The method converges if and only if $p \geqq m$. The rate of convergence predicted by (3) is completely confirmed by computational experiment, and justifies the increase from Courant's value of $p=1$.

There are other questions in the convergence theory of finite elements, and we summarize very briefly two of the most fundamental. ${ }^{4}$

1. The rate of convergence in other norms. It is important to estimate the error $u-u_{S}$, not only in the energy norm, but also in $L_{2}$ or in the maximum norm. In $L_{2}$, Aubin and Nitsche independently discovered an argument based on duality (known as the Nitsche trick) which gives the correct estimate

$$
\left\|u-u_{S}\right\|_{0}=O\left(h^{p+1}+h^{2(p+1-m)}\right) .
$$

Pointwise estimates, on the other hand, are not at all easy; they are much

\footnotetext{
3 The key lemma is due to Bramble and Hilbert.

${ }^{4} \mathrm{I}$ hope the book [9] will serve as a general reference to the theory, and to the mathematical and engineering literatures on finite elements. The subject has grown at a nearly absurd rate, and it looks as if convergence theorems can be proved almost at will. Perhaps that reflects not only the simplicity, but also the "rightness," of the underlying ideas.
} 
less natural for variational problems, and except for regular meshes the problem is still open.

2. Nonconforming elements and numerical integration. Both of these involve variational crimes; the basic rules of the Ritz process are broken, and the projection property (1) no longer holds. In the nonconforming case the trial functions have infinite energy, $a(v, v)=\infty$, because of the way the polynomials are pieced together. To compensate, the energy is changed to a sum of integrals over the separate triangles, and the discontinuities at the boundaries are ignored. In numerical integration (which is standard in the finite element subroutines for calculating $K$ and $F$ ) the underlying functional is altered in a different way - to a discrete sum. In the example of Poisson's equation, the functional which is actually minimized is

$$
\sum w_{i}\left(v_{x}^{2}+v_{y}^{2}-2 f v\right)\left(\xi_{i}\right) .
$$

The weights $w_{i}$ and evaluation points $\xi_{i}$ come from a quadrature formula.

In both cases, convergence is no longer an automatic consequence of the approximation properties of $S$. There is an extra test for the consistency of the discrete problem, and, in fact, nonconforming elements usually fail this test; convergence becomes the exception rather than the rule. The test is this: around an arbitrary patch of elements, impose boundary conditions in such a way that the true solution $u$ is a polynomial of degree $m$, and look to see whether $u_{s}$ coincides identically with $u$. (All polynomials of degree $m$ are present in $S$, by the earlier necessary condition $p \geqq m$.) This patch test was devised by Irons, and for a large class of problems [2], [7] it implies convergence of order $h$ in the energy norm. There is also a higher-order patch test for the more rapid convergence which goes with more accurate numerical integration.

EXAMPLE. Consider the space of piecewise linear functions which are constrained only by continuity at the midpoints of the edges between triangles. These midpoints become the nodes and the space is three times larger than Courant's. It is much more useful in applications to fluid dynamics, where the constraint of zero divergence annihilates Courant's space. Nevertheless, the space is nonconforming-there is a jump in $v$ which varies linearly along the edge and vanishes at the midpoint. This property turns out to be the key to the patch test. After an application of Green's theorem [9], success in the test is assured if along each edge, the integral of the jump is zero. A linear function which vanishes at the middle of its domain does have zero integral, so that in spite of its illegitimate construction, the nonconforming $u_{S}$ will converge to $u$.

The piecewise polynomial spaces $S_{p}^{q}$. There is a natural question about piecewise polynomials which apparently has never been asked or 
answered. It concerns the polynomials of degree $p$ and continuity class $C^{q}$ on a given triangulation (or on a given simplicial complex in $R^{n}$ ). What is the dimension of this space $S_{p}^{q}$, and what is a convenient basis?

In two variables, only the case $q=0$ is understood; $S_{1}^{0}$ is Courant's space, and the quadratic and cubic spaces $S_{2}^{0}$ and $S_{3}^{0}$ were described earlier. $S_{p}^{0}$ is a finite element space with $(p+1)(p+2) / 2$ nodes $z_{j}$ distributed uniformly over each triangle, at the points with barycentric coordinates $(i / p, j / p, k / p)$. For every node there is a basis function, determined by $\varphi_{j}\left(z_{i}\right)=\delta_{i j}$. If there are $V$ vertices, the dimension is essentially $p^{2} V$.

In one variable the question would be comparatively simple. Over each subinterval the polynomial has $p+1$ coefficients, with $q+1$ continuity constraints imposed at the joints. Therefore the dimension is $(p-q) V$, again excluding correction terms from the extreme endpoints. The case $q=p-1$ represents Schoenberg's splines, and illustrates how an increase in continuity spreads the support of the basis functions: the so-called " $B$-splines" are supported over $p+1$ subintervals and the basis is no longer local. This produces serious difficulties at the boundaries of a domain, and explains why splines are not much used in finite element computations. We mention that on a square rather than a triangular mesh in the plane, the question remains simple: the space is a tensor product of one-dimensional spaces and its dimension is $(p-q)^{2} V$.

The first interesting and unresolved case is that of piecewise cubics of class $C^{1}$. We propose to compute its dimension heuristically, as follows. There are ten parameters in the cubic over each triangle, or $10 T$ altogether. Across each edge we need four constraints to assure continuity, and three more for continuity of the normal derivative (which is a quadratic). This produces $7 E$ constraints, but they are not independent. Around any vertex, the quantities $v, v_{x}$, and $v_{y}$ are now certain to be continuous between each triangle and the next. But then continuity between the last triangle and the first, as we circle the vertex, is a redundant constraint-of which there are $3 \mathrm{~V}$. Therefore the total number of free parameters is

$$
10 T-7 E+3 V=20 V-21 V+3 V=2 V .
$$

This we conjecture to be the dimension of $S_{3}^{1}$; we have no ideas about its basis.

REMARK. A similar calculation for $C^{1}$ piecewise quadratics suggests that there are none. Powell has convinced us, however, that on a special triangulation (a square mesh, with all diagonals drawn in) there do exist such quadratics with compact support. Apparently, with this triangulation, there is an extra redundancy of the constraints.

Our questions about $S_{p}^{q}$ can be made simpler and more precise in the 
following way. Let the unit square be divided into $N^{2}$ small squares, and then into $2 N^{2}$ triangles by the diagonals of slope +1 . We impose periodicity at the outer boundaries of the large square, so that each $v$ in $S_{p}^{q}$ can be extended to a doubly 1-periodic function on the whole plane. Then, not asymptotically but exactly, there are $N^{2}$ vertices, $2 N^{2}$ triangles, and $3 N^{2}$ edges. On this triangulation we can count the parameters, constraints, and redundancies, with the following result:

Conjecture. For $p>2 q, \operatorname{dim} S_{p}^{q}=(p-q)(p-2 q) N^{2}$.

In the case $p=3, q=1$, this reproduces the $2 V$ computed earlier. And for the spaces $S_{p}^{0}$, the dimension $p^{2} V$ is correct.

We also believe that there should exist $M=(p-q)(p-2 q)$ functions $\psi_{i}$, whose translates

$$
\psi_{i j k}(x, y)=\psi_{i}(x-j / N, y-k / N), \quad j, k=1, \ldots, N,
$$

form a basis for $S_{p}^{q}$. Like the splines in one variable, these $\psi_{i}$ will be supported over a number of adjacent triangles, probably too many for the existence of a nodal (finite element) basis.

In this form the problem invites a Fourier transformation. There is translation invariance, and the degree of continuity which is awkward to determine at edges and vertices of triangles becomes obvious from the Fourier transform. Furthermore, these transforms are not impossibly complicated; they are ratios of exponential polynomials to polynomials. But the actual construction of the $\psi_{i}$ is genuinely an open problem.

\section{REFERENCES}

1. R. Courant, Variational methods for the solution of problems of equilibrium and vibration, Bull. Amer. Math. Soc. 49 (1943), 1-23, MR 4, 200.

2. M. Crouzeix and P. A. Raviart, Conforming and nonconforming finite element methods for solving the stationary Stokes equations (to appear).

3. G. Pólya, Sur une interprétation de la méthode des différences finies qui peut fournir des bornes supérieures ou inférieures, C. R. Acad. Sci. Paris 235 (1952), 995-997. MR 14, 656.

4. - Estimates for eigenvalues, Studies in Mathematics and Mechanics Presented to Richard von Mises, Academic Press, New York, 1954, pp. 200-207. MR 16, 482.

5. I. J. Schoenberg, Contributions to the problem of approximation of equidistant data by analytic functions. Part A. On the problem of smoothing or graduation. A first class of analytic approximation formulae, Quart. Appl. Math. 4 (1946), 45-99. MR 7, 487.

6. G. Strang, Approximation in the finite element method, Numer. Math. 19 (1972), 81-98.

7. - Variational crimes in the finite element method, Mathematical Foundations of the Finite Element Method, Academic Press, New York, 1972.

8. G. Strang and A. Berger, Change of solution due to change of domain, Proc. Sympos. Pure Math., vol. 23, Amer. Math. Soc., Providence, R.I., (to appear).

9. G. Strang and G. Fix, An analysis of the finite element method, Prentice-Hall, Englewood Cliffs, N.J., 1973.

10. J. L. Synge, Triangularization in the hypercircle method for plane problems, Proc. Roy. Irish Acad. Sect. 54 A (1952), 341-367. MR 14, 801.

11. W. T. Tutte, A census of planar triangulations, Canad. J. Math. 14 (1962), 21-38. MR 24 \# A695. 